\title{
PÉRDIDA DE DERIVADOS SANGUÍNEOS Recomendaciones medicoquirúrgicas para disminuir su uso
}

Cesar Eduardo Jiménez Jiménez MD. *

\section{Resumen}

El uso de los derivados sanguíneos ha venido disminuyendo en la práctica médica dado el aumento de enfermedades transmitidas por las transfusiones y el conocimiento claro de los médicos al considerar la transfusión como un verdadero transplante con todas sus complicaciones. Los cirujanos nos vemos enfrentados a la negativa de algunos pacientes para recibir derivados sanguíneos por convicciones personales o religiosas, como es el caso de los Testigos de Jehová.

Existen muchas medidas para disminuir el uso y la pérdida de sangre en cirugía, que abarca desde una buena técnica quirúrgica, pasando por el uso de sustancias que favorezcan el volumen de glóbulos rojos, como la eritropoyetina y el hierro, sustancias antifibrinolíticas como los análogos de la lisina, medidas intraoperatorias para disminuir la pérdida de sangre, como el salvador de células y técnicas anestésicas con el mismo fin. En este artículo se presenta un paciente Testigo de Jehová, manejado con algunas de estas medidas y se hace una revisión sobre las recomendaciones actuales en prácticas transfusionales avanzadas.

Palabras clave: Testigos de Jehová, eritropoyetina, salvador de células, transfusión, desmopresina.

\section{Introducción}

Desde el descubrimiento de los grupos sanguíneos por Landsteiner en 1900, se han realizado grandes adelantos en la medicina transfusional, incluida la aparición de los bancos de sangre en las décadas de 1940 y 1950, haciendo día a día la transfusión un procedimiento más seguro. Sin embargo, los riesgos siempre estarán presentes y en algunos casos mortales. El estudio de la sangre para evitar el contagio de enfermedades nunca es perfecto. Se pueden transmitir virus como el de la inmunodeficiencia humana (HIV) y la hepatitis $\mathrm{C}$ $\mathrm{y}$, como se informó hace poco, la enfermedad de Creutzfeld Jacob transmitida por priones. Es posible que se descubran otros patógenos sanguíneos que en la actualidad no conozcamos.

Cirujano general. Departamento de Cirugía, Hospital de San José y Clínica del Occidente. Miembro del Comité Médico de enlace para los Testigos de Jehová.
Los pacientes sometidos a transfusiones desarrollan inmunosupresión y aumento en la incidencia de infecciones nosocomiales (neumonías e infecciones de herida quirúrgica), en especial cuando la sangre 1leva almacenada más de 14 días. ${ }^{1,2}$

La conservación de productos sanguíneos es un concepto global que incluye todas las estrategias médicas, quirúrgicas y farmacológicas para disminuir la pérdida de sangre durante la cirugía y el uso de transfusiones; es así como existen numerosas técnicas que han probado ser eficaces y otras no; se pueden utilizar varias al mismo tiempo dependiendo de las necesidades del paciente. Estas alternativas al manejo de productos sanguíneos representan una manera diferente y avanzada de tratar estos pacientes, por lo que algunos autores han denominado este campo como prácticas transfusionales avanzadas ${ }^{(1,2)}$ creándose en muchos sitios del mundo la llamada medicina o cirugía sin sangre, con centros especializados para tratar pacientes sin usar productos sanguíneos. ${ }^{2-4}$ 
Las prácticas transfusionales avanzadas, se basan en tres pilares: ${ }^{1,2,5}$

- Incremento de la masa de glóbulos rojos.

- Disminución de la pérdida de sangre perioperatoria.

- Optimización de las técnicas transfusionales.

Los esfuerzos para disminuir el uso de productos sanguíneos ha sido liderado por la comunidad de Testigos de Jehová y por las personas que son conscientes de los peligros de las transfusiones y los altos costos que pueden representar. 5,6

\section{Presentación de un caso}

J.C. es un paciente de 24 años, testigo de Jehová, que consulta por dolor crónico en miembro superior derecho de seis meses de evolución, manejado primero con analgésicos, sin mejoría; se toma placa de hombro que muestra deformidad de la cabeza del húmero y resonancia nuclear compatible con sarcoma óseo del húmero; se toma biopsia que demuestra osteosarcoma y se programa para resección del tumor en monobloque con preservación de la extremidad.

El paciente entra al programa de cirugía sin sangre de la Clínica del Occidente y se inicia manejo con eritropoyetina y hierro parenteral. Los exámenes iniciales muestran transferrina $180 \mathrm{ng} / \mathrm{ml}$ y saturación de transferrina del $18 \%$, cuadro hemático con 6.700 leucocitos, hematócrito 36,1, hemoglobina 13,1 g/dl y volumen corpuscular medio de 91,6.

Se inició esquema con eritropoyetina $300 \mu / \mathrm{k} /$ día al primero, cuarto, séptimo y décimo días antes del procedimiento, previa hospitalización para administrar $300 \mathrm{mg}$. de hierro parenteral intravenoso. El control al séptimo día fue 7.200 leucocitos, hemoglobina 16 , hematócrito 47 e índice reticulocitario de 3,4. No se desarrollaron complicaciones durante este período.

Se llevó a cirugía y se practicó hemodilución normovolémica aguda en la inducción anestésica, obteniendo $1.500 \mathrm{cc}$ de sangre que se reemplazaron con $500 \mathrm{cc}$ de coloides y $1.000 \mathrm{cc}$ de cristaloides $\sin$ complicaciones. Se realizó hipotensión controlada con dosis bajas de nitroglicerina en infusión, posición de Fowler y control vascular minucioso, procedimiento sin complicaciones. Hubo estabilidad hemodinámica durante todo el transoperatorio, se reinfundieron unidades extraídas a las tres horas del procedimiento. El tiempo total de la cirugía fue de cinco horas 35 minutos, se preservó la extremidad del paciente y se implantó prótesis. El paciente evolucionó de manera satisfactoria con cuadro hemático a las 12 horas que mostró hemoglobina $11 \mathrm{~g} / \mathrm{dl}$ y hematócrito 31 . Se dió salida con suplencia de sulfato ferroso $300 \mathrm{mg}$ por vía oral cada 12 horas durante tres meses.

\section{Los Testigos de Jehová}

Son miembros de una fe cristiana establecida en 1870 en Pittsburgh, Pensilvania, Estados Unidos; existen seis millones de practicantes a través del mundo, en Colombia son cerca de 120.000 personas 3,5 y es una de las comunidades religiosas con mayor crecimiento anual. De acuerdo con su interpretación de la Biblia, se niegan a recibir transfusiones, ejerciendo su libertad de culto y libre albedrío. El texto hace más de 400 referencias con respecto a la sangre y a veces la conecta con la salvación de la vida.

Los Testigos de Jehová creen que la Biblia es la palabra divina de Dios, por lo tanto la guía para su vida. Uno de estos principios es que la sangre una vez ha sido removida del cuerpo debe desecharse y no debe ser reinfundida; la interpretación que se da para no recibir transfusiones de sangre se basa en ciertas partes del antiguo y nuevo testamento, como son:

Levítico 17:10,11 "Cualquier hombre de la casa de Israel o de los extranjeros que moran entre ellos, que comiere cualquier clase de sangre, Yo me volveré contra el que come la sangre, y le exterminaré de entre su pueblo, porque la vida de la carne está en la sangre". ${ }^{5,7}$

Levítico 17:13,14 "No comeréis la sangre de ninguna carne, porque la vida de toda carne es su sangre, cualquiera que la comiere será exterminado" 5,7 
Deuteronomio 12:23,25 "La sangre es la vida; así que no deben comer la vida junto con la carne. Lo que deben hacer es derramarla en la tierra como agua. No la coman, y les irá bien a ustedes y a sus hijos por hacer lo recto" 5,7

Otros son Génesis 9:3,5 y Hechos de los Apóstoles 15: 19,20. ${ }^{5,7}$ Como es natural, en los tiempos en que se escribió la Biblia no se conocía el concepto de transfusión (el cual se describió por primera vez en el siglo XVI, por el profesor de anatomía Thomas Bartholin), pero la interpretación de los Testigos de Jehová es que a partir de las referencias anteriores, no se debe comer sangre, que se equipara a la no utilización de productos sanguíneos; no se debe almacenar su sangre de ningún modo ni tampoco debe interrumpirse el flujo continuo de sangre.

Cuando un paciente se niega a recibir productos hemáticos, están implicados los siguientes principios éticos y derechos fundamentales. ${ }^{5,8-10}$

Principio de autonomía: El paciente tiene derecho a participar en las decisiones que se tomen sobre su enfermedad, y aquí entra a jugar el consentimiento informado que garantiza a los pacientes el uso de sus libertades. Para que se ejerza la autonomía, la decisión del paciente se tomará con conocimiento de la actuación médica que se proponga, de su significado, de las consecuencias que de ellas puedan derivarse y debe tomarse en ausencia de coacciones externas a la persona.

Principio de beneficencia: hacer el bien ante todo y prevenir el daño. En la antigüedad era el principio rector de la práctica médica, pero hoy se debe asociar con los principios personales y deseos del paciente, estén o no en concordancia con los del médico.

Principio de no maleficencia: traduce el primum non nocere, primero no hacer daño. Inyectar productos sanguíneos a un paciente Testigo de Jehová sin su consentimiento puede considerarse un daño moral. Cuando se transgrede la decisión de no usar productos sanguíneos, se ve afectada su concepción religiosa, su visión espiritual acerca de la vida eterna y el desarrollo de su relación con Dios.
Por lo general los médicos difieren de sus pacientes en los intereses, objetivos, experiencia, valores, estilos de vida y manera de abordar diferentes situaciones, en especial la enfermedad, lo que genera tensión en la relación médico paciente. Cuando el galeno determina qué es mejor para el paciente, tiende a exponer razones fisiológicas para su tratamiento, mientras que los pacientes involucran razones sentimentales, sociales, espirituales y psicológicas, de tal manera que la recomendación puede no ser aceptada por el paciente. Para una buena relación, el profesional debe aceptar la autonomía del enfermo en las decisiones sobre su salud, aceptándolas, así sean contrarias a la directriz médica.

En casos no urgentes los médicos pueden abstenerse de atenderlo cuando no acepta sus recomendaciones, sin ninguna implicación medicolegal; la mejor conducta es ayudar al paciente a encontrar un colega que acepte su negativa a los productos sanguíneos.

Cuando un cirujano o cualquier médico se enfrenta a un Testigo de Jehová, debe existir una comunicación abierta y honesta, respetando ante todo su libertad de decisión como persona y paciente, explicar con claridad el procedimiento quirúrgico que necesite y la importancia del uso de productos sanguíneos; si rechaza esa terapéutica, debe remitirse a otro especialista que tenga experiencia en el manejo de programas de medicina sin sangre y contactar al comité de enlace médico de los Testigos de Jehová.

La prohibición aplica a la sangre total, glóbulos rojos empaquetados, células blancas, plasma y plaquetas, pero no a fracciones de sangre mínimas como: inmunoglobulinas, albúmina, eritropoyetina y factores de coagulación. Los testigos de Jehová son autónomos para decidir el uso de estas últimas.

El uso de vacunas de fuente no sanguínea, transplante de órganos o tejidos, diálisis y circulación extracorpórea, son de libre escogencia mientras la 
máquina no esté cebada con sangre sino con cristaloides, coloides o sangre extraída del paciente conectada a un circuito el cual no interrumpa la circulación (salvadores de células, hemodilución normovolémica). Todas estas medidas deben discutirse en forma amplia por el médico tratante antes de instaurar cualquier tratamiento y se debe dejar escrito el deseo del paciente y los procedimientos que autoriza y los que no.

\section{Aumento del volumen de glóbulos rojos}

Uso de hierro y eritropoyetina (EPO): la EPO es una hormona glucoproteíca producida por el riñón, a nivel del mesangio, cuya función es aumentar la producción de glóbulos rojos en la médula ósea, incrementando la diferenciación y proliferación de precursores eritroides. El principal estímulo para la liberación de EPO es la hipoxia, que aumenta la expresión del gen de la EPO. Los niveles normales oscilan entre 4 y $30 \mathrm{mu} / \mathrm{ml}$, y los niveles aumentan en la medida en que la hipoxia sea más severa. La EPO se obtiene por recombinación genética, siendo exactamente igual a la EPO humana endógena y se viene utilizando desde 1986. Es un polipéptido glucosilado con 166 aminoácidos y un peso molecular de 34.000 daltons. ${ }^{11-13}$

La EPO, se indica en diferentes situaciones clínicas:

- Anemia de los pacientes con insuficiencia renal crónica.

- Prevención de la anemia en los prematuros con peso entre 750 y $1.500 \mathrm{~g}$ al nacer y una edad gestacional menor de 34 semanas.

- Prevención y tratamiento de la anemia en pacientes adultos con tumores sólidos sometidos a quimioterapia con cisplatino.

- Tratamiento de la anemia en pacientes adultos con mieloma múltiple, linfoma no Hodking de bajo grado o leucemia linfocítica crónica.
- Para aumentar el rendimiento de la sangre autológa en los programas de autotransfusión.

- Para aumentar la masa eritrocitaria en los testigos de Jehová en la etapa prequirúrgica (esta indicación se explicará más a fondo en el artículo).

- Anemia de los enfermos con SIDA.

- Anemia en enfermedades crónicas (artritis reumatoidea, infección crónica).

Contraindicaciones de la EPO:

- Hipertensión arterial no controlada.

- Hipersensibilidad al principio activo.

- Primer trimestre del embarazo.

- Antecedente de evento coronario, cerebrovascular o episodios tromboembólicos en el último mes (para los que están en programa de autotransfusión).

El objetivo de la EPO en los testigos de Jehová es aumentar la masa de glóbulos rojos hasta un nivel de hematócrito de 42 a 45 para resistir una mayor pérdida de sangre durante la cirugía y evitar el uso de transfusiones. Además, se utiliza para optimizar la hemodilución normovolémica prequirúrgica en los pacientes que la acepten. ${ }^{3,6,9,12,13}$

Existen muchos esquemas para utilizar la EPO y aumentar la masa eritrocitaria prequirúrgica, entre los más conocidos tenemos: ${ }^{(12,13,14,15,16)}$

- $300 \mu / \mathrm{kg}$ los días 1 , 4, 7 y 10 antes de la cirugía, vía subcutánea (SC).

- $400 \mu / \mathrm{kg}$ los días $1,5,9$ antes de la cirugía, SC.

- $600 \mu / \mathrm{kg}$ los días 1 y 10 antes de la cirugía, SC.

- $600 \mu / \mathrm{kg}$ dos veces por semana, tres semanas antes de la cirugía, SC. 
- $600 \mu / \mathrm{kg}$ los días 7, 14, 21 antes de la cirugía.

- 300-500 $\mu / \mathrm{kg}$ nueve días antes, el día de la cirugía y cuatro días después.

- $300 \mu / \mathrm{kg}$ diarios durante cuatro días antes de la cirugía y $150 \mu / \mathrm{kg}$ diarios durante los siguientes siete días a partir del postoperatorio.

- $300 \mu / \mathrm{kg}$ por cinco días antes y $150 \mu / \mathrm{kg}$. los dos días siguientes a la cirugía.

- $600 \mu / \mathrm{kg}$ una vez por semana durante tres semanas antes de la cirugía.

Complicaciones de la terapia con EPO: algunos pueden desarrollar hipertensión arterial leve, que se maneja con hipotensores convencionales, pero si persiste debe suspenderse la EPO. Los eventos tromboembólicos son raros, oscilando entre $2 \%$ y $3 \%$, pero pueden aumentar en los pacientes con factores de riesgo, como falla cardíaca, mayores de 65 años o antecedentes de coagulopatías.

Es importante anotar que la EPO no es la única medida para una eritropoyesis efectiva; la suplencia de hierro, vitamina $\mathrm{C}$, ácido fólico y vitamina B12 es crucial.

Independiente del esquema usado para administrar la EPO, la ferritina basal es el determinante más importante en la respuesta que pueda generar la EPO en un paciente.

Estudios han demostrado que aquellos con depósitos bajos o normales de hierro, generan eritropoyesis inadecuada cuando se suministra EPO, manifestándose por aumento de la masa eritrocitaria pero dando lugar a eritrocitos hipocrómicos y en algunos casos microcíticos, lo que se denomina eritropoyesis ineficaz ya que la cantidad de hierro existente no suple las necesidades de las nuevas células en nacimiento; por lo tanto, a todo paciente que vaya a ser sometido a terapia con EPO, se le debe determinar los niveles de ferritina sérica, transferrina y cuadro hemático con diferencial mediante técnica automatizada.
Los que tengan ferritina menor a $200 \mathrm{ng} / \mathrm{ml} \mathrm{o}$ una saturación de transferrina menor al $20 \%$ se les suministra hierro parenteral, no oral. ${ }^{1,2,14-18} \mathrm{La}$ dosis se calcula con la siguiente fórmula: peso $\mathbf{~} \mathbf{g} \mathbf{x}$ [ normal de $\mathbf{H b}-\mathbf{H b}$ actual en $\mathrm{g} / \mathrm{dl}$ ] x 0,24 + 500 mg. La vía intravenosa repone las reservas con mayor rapidez y eficacia que la oral o intramuscular.

Existen diferentes presentaciones parenterales, pero la más usada es el hierro sacarosa o Venofer. Es importante recalcar que es un medicamento que puede generar reacciones anafilácticas severas que pueden llevar a la muerte. Se debe aplicar siempre diluido en solución salina normal y en infusión continua de manera lenta, vigilado por un médico.

Para los pacientes con niveles normales de hierro basal, se recomienda la vía oral, en dosis de $300 \mathrm{mg}$ de hierro elemental al día, mínimo durante un mes.

El control de la terapia con EPO y hierro debe controlarse cada semana con índice de reticulocitos y cuadro hemático automatizado. Se recomienda el uso de vitamina $\mathrm{C}$, porque el ácido ascórbico facilita la incorporación del hierro en la protoporfirina y mejora la respuesta a la EPO (500 mg vía oral cada 8 horas).

\section{Disminución de la pérdida de sangre}

Es muy importante que todo el equipo que participa en el manejo de los Testigos de Jehová, esté consciente y acepte el rechazo del enfermo a los productos sanguíneos. El manejo se inicia desde en el momento en que consulta a una institución con programas sin sangre. La primera valoración preanestésica la hace el cirujano tratante, explicando el procedimiento quirúrgico y estableciendo las técnicas de ahorro de sangre que el paciente acepte, consignándolas por escrito en consentimientos informados y en la historia clínica. Existen diferentes medidas durante el perioperatorio para disminuir la perdida de sangre cuya responsabilidad es del cirujano y del anestesiólogo, como son las técnicas pre, peri y postquirúrgicas, las anestésicas, las 
medidas farmacológicas, el salvamiento de células sanguíneas y los transportadores artificiales de oxígeno.

Las técnicas prequirúrgicas ${ }^{19-21}$ indicadas son:

- El número de punciones que se realicen a un testigo de Jehová deben ser las estrictamente necesarias, utilizando técnicas de micromuestra para disminuir la pérdida de sangre.

- Luego de cualquier punción o retiro de catéter, se debe hacer presión prolongada y asegurarse que el sangrado se detenga.

- Disponer de personal médico y paramédico con experiencia en cirugía sin sangre y pacientes Testigos de Jehová.

- Todo paciente Testigo de Jehová debe llevar un brazalete que informe su negativa a productos sanguíneos y una nota en la historia clínica al alcance de la vista del personal que la manipule. Además, todos los Testigos de Jehová portan de manera permanente una carta de poder para atención médica, en donde estipulan su negativa a la sangre y sus principales componentes y mencionan las alternativas aceptables, exoneran al personal que los atienda de toda responsabilidad por cualquier daño que pudiera resultar de su decisión a pesar del cuidado competente que reciban en el resto de atención.

- Mantener el volumen intravascular con coloides o cristaloides todo el tiempo, asegurando un adecuado volumen circulante efectivo.

- Elaborar una historia clínica completa y detallada sobre antecedentes personales y familiares con trastornos hemorrágicos e identificar medicaciones que puedan alterar la hemostasia (warfarina, heparina, medicamentos homeopáticos, etc).

- Planear el procedimiento quirúrgico y realizarlo en el menor tiempo posible por un cirujano experto y hábil.
- En los pacientes con tumores sólidos, se recomienda la embolización prequirúrgica para disminuir el tamaño tumoral y el sangrado intraoperatorio.

Las técnicas intraquirúrgicas ${ }^{22-26}$ recomendadas son:

- Seguir los principios quirúrgicos Halstedianos (disección por planos, buen trato a los tejidos, hemostasia meticulosa con ligaduras selectivas y no en masa).

- Uso de dispositivos que disminuyan el sangrado, como el electrocauterio, el láser de argón, cirugía endoscópica o video asistida, escalpelo ultrasónico o de radiofrecuencia, pegantes de fibrina y sustancias procoagulantes tópicas.

- Las técnicas de cirugía de control de daños para trauma son procedimientos que minimizan la pérdida de sangre y se deben considerar en estos casos.

- Para minimizar la pérdida de sangre por la fuerza de gravedad, se recomiendo elevar la zona a intervenir por encima del nivel de la aurícula derecha, lo que disminuye la presión venosa hidrostática y a su vez el sangrado intraoperatorio, por ejemplo en varicectomías al colocar en posición de Trendelemburg. Las cirugías de columna lumbosacra que requieran decúbito prono, se aconseja colocar dos rollos longitudinales a lo largo de ambas líneas medioclaviculares ya que dicha posición sin soporte genera incremento de la presión intrabdominal y de la cava inferior con aumento retrógrado en las venas lumbares, que genera mayor sangrado intraoperatorio. Mantener un volumen circulante efectivo con la menor cantidad de líquidos intravenosos, como lo ha demostrado la experiencia en cirugía hepática y de trauma que la sobrehidratación aumenta el sangrado al elevar las presiones venosas y arteriales; debe mantenerse una presión arterial media de $90 \mathrm{~mm}$ $\mathrm{Hg}$ y una presión venosa central igual o menor a $5 \mathrm{~cm}$ de agua. 
- Uso de torniquetes en cirugías de extremidades para minimizar la pérdida sanguínea durante la disección.

- Uso de compresas o campos en los bordes de la herida impregnados con adrenalina, en una solución de una parte por millón, para disminuir el sangrado por allí.

- Hipotermia, aconsejada en neurocirugía y cirugía cardíaca para disminuir el consumo de oxigeno y proteger contra la isquemia. El objetivo de la hipotermia es bajar la temperatura a 30-32 grados centígrados, para disminuir el consumo de oxigeno en un $48 \%$, generar una desviación de la curva de disociación de la oxihemoglobina a la izquierda, lo que aumenta la afinidad de la hemoglobina por el oxígeno y disminuye la liberación tisular, sin generarse hipoxia; la hipotermia aumenta la viscosidad sanguínea pero si se acompaña de hemodilución normovolémica, se contrarresta este fenómeno.

Es importante recalcar que la hipotermia no controlada es un factor muy importante que genera coagulopatía. La experiencia en cirugía de control de daños ha demostrado que los pacientes con temperatura menor de 35 grados tiene alteración de todos los procesos de coagulación.

Un litro de cristaloides administrados a temperatura ambiente ( 20 grados) reduce la temperatura de un paciente de $70 \mathrm{~kg}$ en 0,25 grados; de tal manera que todos los líquidos que se apliquen deben tener una temperatura de 40 grados centígrados.

\section{Las técnicas postquirúrgicas ${ }^{24,27-29}$ relevantes} son:

- Reinfusión postoperatoria de sangre recolectada por tubos toráxicos, mediastinales o sistemas de drenaje en cirugía ortopédica.

- Estar muy atento a signos de resangrado e implementar rápido medidas diagnósticas y terapéuticas para detectarlos y solucionarlos.
- Uso de técnicas de cirugía de control de daños (empaquetamientos, embolizaciones selectivas, etc.).

- Profilaxis antitrombótica.

- Profilaxis con inhibidores de la bomba de protones o protectores de mucosa gástrica, para evitar las úlceras por estrés.

- Sedación y relajación adecuada, analgesia suficiente para disminuir el consumo y demanda de oxígeno.

Las técnicas anestésicas incluyen la hipotensión controlada ${ }^{30-32}$ y la anestesia regional. ${ }^{33-36}$

La primera se define como la disminución de la tensión arterial sistólica a 80 o $90 \mathrm{mmHg}$ o una reducción de la presión arterial media entre 50 y 55 $\mathrm{mmHg}$ en un paciente previamente normotenso. Se recomienda esta técnica en intervenciones en órganos como el corazón, sistema nervioso central, riñones, vísceras abdominales y ojos; no se aconseja para procedimientos que requieran anastomosis vasculares o la transposición de tejidos bien perfundidos como flaps o injertos.

La hipotensión se logra con medicamentos como la nitroglicerina, el halotano o isoflurano, anestesia peridural o raquídea, óxido nitroso, diltiazem, trimetafán (bloqueador ganglionar), nicardipina o nitroprusiato de sodio. La isquemia miocárdica, cerebral u óptica son complicaciones potenciales con esta técnica. Las contraindicaciones de la hipotensión controlada, son:

- Enfermedad cerebrovascular.

- Alteración cardiovascular (enfermedad coronaria, estenosis aórtica, hipertensión arterial no controlada, falla cardíaca.

- Enfermedad pulmonar severa.

- Falla renal.

- Falla hepática. 
- Hipovolemia.

- Anemia severa.

- Embarazo.

- Incapacidad para monitorizar adecuadamente a un paciente.

Usando técnicas anestésicas regionales como los bloqueos, anestesia espinal o peridural, se ha visto una disminución en la pérdida sanguínea del 30\% a 40\%, comparada con la anestesia general. Los mecanismos que la explican, son:

- Disminución de la presión arterial con bloqueo simpático.

- Disminución de la presión venosa en los sitios anestesiados regionalmente.

- La anestesia general al utilizar ventilación mecánica controlada con presiones positivas intermitentes, genera un aumento de la presión auricular media, mayor que en anestesia regional.

En cuanto a las medidas farmacológicas, existen diferentes medicamentos que disminuyen el sangrado antes, durante y después del procedimiento quirúrgico; algunos han probado su eficacia y otros están siendo evaluados para conocer su beneficio real. Entre ellos tenemos la desmopresina que es un análogo de la vasopresina u hormona antidiurética. Induce la liberación de grandes cantidades de factor de Von Willebrand, lo que aumenta la adherencia plaquetaria al endotelio y se usa a una dosis de 0,3 microgramos por kilo de peso diluida en $50 \mathrm{cc}$ de solución salina normal al $0,9 \%$, se infunde en 15 a 30 minutos, antes de la incisión quirúrgica. La corrección del tiempo de sangría ocurre entre 30 minutos y 2 horas después de la aplicación, con duración de 8 horas. Se recomienda especialmente en pacientes con trastornos plaquetarios, como aquellos que toman aspirina y en insuficiencia renal crónica, enfermedad de Von Willebrand, síndromes mielodisplásicos y cirrosis. El 30\% desarrollan hipotensión por causa desconocida, por lo que se recomienda la infusión lenta en 20 minutos ${ }^{1,2,37}$

Los análogos de la lisina son los compuestos más usados para hemostasia en cirugía cardíaca en los Estados Unidos. La unión del plasminógeno a la fibrina ocurre gracias a un sitio dependiente de lisina en la molécula de plasminógeno. Los análogos de este aminoácido bloquean el sitio y se impide la lisis de la fibrina. Los dos representantes de este grupo, son los ácidos tranexámico y epsilón aminocaproico. Basados en la literatura disponible, el uso del primero a $10 \mathrm{mg} / \mathrm{kg} / \mathrm{h}$ disminuye los volúmenes de drenaje por tubos en cirugía cardíaca en un 25 a 30\%, comparado con el uso de placebos. A pesar de la reducción en los drenajes, los datos disponibles concluyen que los análogos de la lisina no disminuyen la rata de reexploración por sangrado postcirugía cardiovascular o hepática. 1,2,38,39

Existen diferentes proteasas en el organismo (antitrombina III, la alfa 2 antiplasmina, alfa 1 antiquimiotripsina, alfa 2 microglobulina) que ejercen acciones enzimáticas en el tracto gastrointestinal, sistema respiratorio, aparato circulatorio, etc. Los inhibidores de estas proteasas, son inhibidores suicidas ya que bloquean la proteasa y la antiproteasa; una de estas sustancias es la aprotinina, polipéptido básico (pK 10) de 58 aminoácidos con un peso molecular de 6.512 daltons, es un inhibidor de amplio espectro de proteasas (en especial las que participan en la cascada de la coagulación) que actúa en los residuos de serina de las proteasas; se obtiene del pulmón de bovino y del intestino porcino. El grado de inhibición depende de la dosis de tal manera que para la tripsina se requieren dosis bajas y para la trombina altas. La acción básica de la aprotinina es inhibir la fibrinolisis al inactivar la plasmina libre.

La aprotinina en altas dosis se usó por primera vez en 1987 y existen más de 40 informes en la literatura que hablan de su eficacia, ${ }^{1,2,40-43}$ concluyendo que reduce los drenajes por tubos en cirugía cardíaca, disminuye el número de transfusiones, el 
uso de inotrópicos y de agentes antiarrítmicos en el postoperatorio de cirugía cardíaca. También se ha visto una baja en la necesidad de soporte ventilatorio y menos eventos cerebrovasculares postcirugía cardíaca. Sus efectos benéficos también se han visto en cirugía ortopédica, hepática y vascular.

El salvador de células es la técnica de conservación de sangre más usada hoy en el perioperatorio. Descrito por primera vez en 1886 , en un paciente que sufrió politraumatismo al ser arrollado por un tren, la sangre del paciente perdida durante la cirugía se recolectó en un platón estéril y se trató con fosfato de soda, se filtró en una gasa y se reinfundió por medio de una jeringa. El paciente sobrevivió y el concepto de autotransfusión nació; con el advenimiento de los bancos de sangre en 1940 y 1950 la recuperación de sangre se dejó de usar y solo hasta las décadas de los 70 y 80 se inventaron las primeras máquinas de recuperación de sangre intraoperatorias, gracias a los adelantos en el equipo de circulación extracorpórea. La primera disponible en el comercio fue la unidad Bentley que consistía en una bomba con rodillo, un sistema de aspiración y un recolector, con alta incidencia de embolismo aéreo por lo que se dejó de usar. ${ }^{2,44,45}$

En la actualidad se utilizan diferentes variedades de aparatos salvadores de células que tienen muchas ventajas pues los glóbulos rojos recuperados en la operación no tienen las alteraciones morfológicas de membrana que presentan los de las unidades empacadas provenientes de los bancos de sangre. Además, tienen una capacidad de transporte de oxígeno mayor y una vida media más larga que las unidades almacenadas. Varios estudios han demostrado que en cirugía de aorta abdominal la sobrevida mejora al usar salvador de células. ${ }^{46}$

El concepto actual de recuperación intraoperatoria se basa en el uso de dispositivos que aspiren la sangre del campo quirúrgico a un gradiente no mayor de $80 \mathrm{~mm}$ de $\mathrm{Hg}$ posteriormente es filtrada, lavada, separada, suspendida en una solución salina y por último reinfundida. Se indica en pérdidas de sangre mayores a $1.000 \mathrm{cc}^{44}$
Se usa en trauma, intervenciones abdominales tipo aneurisma de aorta, cirugías hepática, ortopédica (con filtros para espículas óseas), toráxica y neurológica.

Se contraindica cuando hay gran contaminación intestinal de la sangre o con células neoplásicas, aunque hay estudios que muestran que al usar filtros de leucocitos se puede emplear en cáncer. $44,46,47$

Dentro de las complicaciones de la recuperación de células, tenemos:

- Hemólisis y hemoglobinuria, ya que la sangre recuperada tiene niveles muy altos de hemoglobina libre y hay hemólisis por activación del sistema del complemento.

- Embolismo aéreo.

- Diseminación de células tumorales.

- Aspiración inadvertida de productos del campo quirúrgico, tipo gelfoam o surgicell.

- Coagulopatía.

La eficacia del salvamento de células se ha demostrado en varios estudios, dado por la disminución en el uso de sangre alogénica y es una excelente opción en los pacientes Testigos de Jehová. El salvamento de células probablemente causa mayor costo efectivo que el uso de antifibrinolíticos. ${ }^{1,2,44,47}$

En cuanto a los transportadores de oxígeno artificiales la tecnología actual está orientada al desarrollo de sustancias que reemplacen la capacidad de transportar oxígeno sin necesidad de hacer pruebas y que los pacientes acepten libremente, con el fin de utilizarlas para aumentar el volumen intravascular cuando se usa hemodilución normovolémica. Estas sustancias se clasifican en dos tipos:

a) soluciones modificadas de hemoglobina y b) soluciones de perfluorocarbonos (PFC). 
Las primeras son sustancias que desnaturalizan el tetrámero de hemoglobina y utiliza alguna de sus cadenas para el transporte de oxígeno. La más conocida es la alfa diaspirina, que se ha venido usando en los pacientes politraumatizados en la fase prehospitalaria, para aumentar el transporte de oxígeno y lograr un volumen circulante efectivo. 1,2,44

Los PFC son compuestos caracterizados por una alta capacidad de disolución de gases, baja viscosidad, inertes química y biológicamente, pero deben emulsificarse antes de entrar al torrente sanguíneo. Los $\mathrm{PFC}$ se han usado para prevenir la isquemia cardíaca y cerebral, en el manejo del síndrome de reperfusión y en el reemplazo de sangre autóloga, pero aún faltan estudios para definir con certeza su empleo. ${ }^{1,2}$

\section{La optimización en técnicas de autotransfusión} se refiere a la hemodilución normovolémica aguda (HNA) y al programa de autotransfusión.

La HNA se define como la inducción de anemia normovolémica con la extracción de sangre y la restitución de la volemia mediante el suministro de líquidos de reemplazo. No prolonga el tiempo quirúrgico, ni dificulta las funciones del cirujano y además son menores los costos y las incomodidades para el paciente. ${ }^{44}$

La disminución de la masa eritrocitaria incrementa la liberación de oxígeno, aumenta el gasto cardíaco sin elevar la frecuencia por disminución de la poscarga y aumento del retorno venoso (la taquicardia indica sustitución inadecuada de los líquidos de reemplazo), es menor la perdida de glóbulos rojos ya que el paciente está hemodiluido y se reduce el riesgo de trombosis por baja de la viscosidad sanguínea.

Se ha demostrado que la HNA, aumenta en un $200 \%$ el flujo sanguíneo cerebral, muy importante en enfermedad cerebrovascular. ${ }^{1,2,44}$ Sigue siendo controversial el uso de la HNA en enfermedad coronaria, pero se ha visto que con este procedimiento aumenta el gasto cardíaco, disminuye la resistencia vascular coronaria y la viscosidad sanguínea, pudiéndose incrementar el flujo coronario en un $45 \%$ en arterias estenóticas.
La HNA, se debe llevar a un hematócrito límite de $25 \%$. Por debajo de $20 \%$ se inicia la anaerobiosis y aumenta la producción de ácido láctico. Se contraindica en casos de medicación con bloqueadores beta, infección activa, coagulopatías, estenosis aórtica o mitral, angina de pecho inestable, arritmias cardíacas, hipertensión arterial severa, enfermedades crónicas descompensadas como diabetes mellitus, enfermedad pulmonar obstructiva crónica e insuficiencia renal.

La HNA es responsabilidad del anestesiólogo, realizada durante la inducción. La colección de sangre se realiza en bolsas de autotransfusión con anticoagulante y entre cada unidad extraída se debe determinar la hemoglobina y el hematócrito de control. Las flebotomías deben seguir todos los principios de técnica aséptica, se marcan en forma adecuada las unidades extraídas con fecha, hora, identificación del paciente y anestesiólogo responsable. Estas unidades no deben permanecer a temperatura ambiente por mas de seis horas, porque después se consideran contaminadas y deben desecharse. Cuando la reinfusión de la unidad se planea en un lapso de 6 a 24 horas, es necesario refrigerarlas.

Este procedimiento es aceptado por algunos Testigos de Jehová, mientras la sangre extraída no se almacene, sino que permanezca conectada al cuerpo del paciente. El reemplazo de la sangre extraída se puede hacer con coloides o con cristaloides, de acuerdo con los siguientes parámetros: por cada mililitro extraído se debe infundir un mililitro de coloide, y por cada mililitro extraido se deben infundir tres mililitros de cristaloide.

El cálculo del volumen de sangre a extraer, se hace con la siguiente formula:

\section{Vs (Hcti - Hctf)}

\section{Hctm}

cuando Vs:volumen sanguíneo del paciente, Hcti: hematócrito inicial del paciente, Hctf:hematócrito final y Hctm: hematócrito medio. 
El programa de autotransfusión se define como aquel procedimiento mediante el cual un donante o paciente se convierte en receptor de su propia sangre, es aplicable a glóbulos rojos, plasma, plaquetas y progenitores hematopoyéticos.

Se indica en cirugías con pérdidas hemáticas mayores a $1.000 \mathrm{cc}$ o bien $200 \mathrm{cc}$ por hora y en pacientes con grupos sanguíneos escasos, a lo inmunizados o con rastreo de anticuerpos irregulares positivo, así como aquellos que no deseen recibir sangre de otras personas. Tiene las mismas contraindicaciones que la HNA. 1,2,44

El predepósito preoperatorio varía entre una y cinco unidades en un lapso menor de 35 días y el manejo debe ser igual que las de otros donantes, según la ley, de tal manera que se realizan pruebas para VIH, hepatitis, sífilis, enfermedad de Chagas y hemoclasificación directa e inversa.

Entre la extracción de cada unidad debe haber un mínimo de 72 horas y entre la última unidad y la cirugía otras 72. Es importante que estos pacientes tengan suplencia de hierro por vía oral durante el preoperatorio y también de eritropoyetina si es posible. La EPO es de $600 \mu / \mathrm{kg}$ por semana durante tres semanas antes de la cirugía. ${ }^{48}$

\section{Agradecimientos}

Agradezco a la comunidad de testigos de Jehová de Colombia por su apoyo y acompañamiento en el manejo de sus pacientes.

\section{Referencias}

1. Blood conservation and Transfusión alternatives 28th Congress of the International Society of Hematology, $2000 \mathrm{aug}$ 26; Toronto, Canadá. P 5-48

2. Linder van der P. Perioperative blood conservation strategies: an update for clinicians. Can J of Anaesth 2003;.50 (6)

3. Code of practice for the surgical management oj jehovah's witnesses. London: Council of the Royal College of Surgeons of England, 2000. p 1-19; 35-43
4. The New York Center for Bloodless Medicine and sugery, guide. 2003, Br. J. Surg 1998; 75 (4): 44-48.

5. Glenn P. ¿Cómo puede salvarle la vida la sangre? 1990 watch Tower Bible and tract Society Pensilvania. Atalaya 1990; 23-7

6. Man M, Votto J, Kambe J. Managaement of the severely anemic patient who refuses transfusion: lessons learned during the care of a Jehovah's witness. Ann Intern Med 1992; 117: 1042-48.

7. Sagrada Biblia, XXXIX Congreso Eucarístico Internacional de Bogota, 1968.

8. Dixon L. Los testigos de Jehová el desafío quirúrgico JAMA 1981, nov; 246: 2471-72.

9. Dixon L. Transfusiones de sangre: ¿De quien es la decisión? ¿La conciencia de quien debe respetarse? J Med 1988; 88: 463-4.

10. Guzman MF, Morales de MC, Franco E. De la Responsabilidad Civil Médica. Bogotá: Ediciones Rosarista, 1995.

11. Cortes A. Alternativas farmacológicas a las transfusiones de sangre y componentes. Col Médica 1994;25:73-82.

12. Niels Q. Stten B. Birgit W. Carnsten P. Recombinant Human Erythropoietin and Hemoglobin Concentration at Operatio and during the postoperative period: Reduced need for blood transfusions in patients undergoing colorectal surgery- Prospective Double - blind placebo - controlled study. World J Sury 1999; 23: 30-5.

13. Rutherford C, Schneider T, Hilton D, David K. Efficacy of different regimens for recombinant human Erythropoietin in a simulated perisurgical setting: The importance of Iron availability in optimizing response. American J Med 1994; 96

14. Alexander K. Gombotz H. Gootfried F. Subcutaneous Recombinant Human Erythropoietin and autologous blood donation before coronary artery Bypass surgery. Anesthesia Analg 1993; 76: 102-6.

15. Adamson J. Spivak J. Physiologic basis for the pharmacologic use of reombinant human erythropoietin in surgery and cancer treatment. Surgery; Jan1994.

16. Guidelines Consensus Conference: Blood Management Surgical Practice Am J Surg; 1995: 170 (6A).

17. Ramos H, Satoru T, Yoogooo K. Evangelos F Howard D. Liver transplantation withouth the use of blood products. Arch Surg 1994; 129:528-533.

18. Cardemil G, Rodríguez M, Reyes D. Resultados del programa de atención medico-quirúrgica sin uso de sangre no hemoderivados del Hospital Clínico de la Universidad de Chile. Rev Chilena Cirugía 2004; 56 (3): 232-36.

19. MacIntyre A. Blood transfusion and hemostatic management in the perioperative period. Can J Anaesth 1992; 39 (5): Pt 2 R 101-14.

20. Fugh-Bermann A. Herb Drug interactions. Lancet 2002; 355 (9198): 134-38.

21. Faris P. Spende K, Larholt M.O. The predicitive power of baseline hemoglobinfor transfusion risk in surgeey patients. Orthopedics 1999:22 (1) Suppl S 135-40 
22. Torzilli G, Makuuchi K. Inoue E. No mortality liver resection for hepatocelular carcinoma in cirrhotic and noncirrhotic patients; in there a way? A prospective analysis of our approach. Arch Surg 1999; 134 (9): 984-9.

23. Sabiston D,C. Principles of operative surgery: antisepsis, technique sutures and drains; Textbook of surgery ; the Biological Basis of Modern Surgical Practice, 15 th ed. Philadelphia. Saunders, 1997. pp. 253-63.

24. Amours D. Joshipura M, Introduction : Damage control at the start of 21st century. Sugrue M. Injury 2004; 35: suppl 639-41.

25. B,Cherqui R. Safety of selective vascular clamping for major hepatectomies. Malasagne. 1998; 187 (5): 482-86.

26. Schneeberger A, Schulz R. Blood loss in total hip arthroplasty. Lateral position combined with preservation of the capsule versus supine position combined with capsulectomy: Arch Orthop, Trauma Surg 1998; 117 (1-2): 47-9.

27. Postsplenectomy blood salvage in anemic patient; De Rai P.; Rebulla H.; JAMA, 258, N. 10 (1987),1332.

28. Evaluation of autologous she blood for autotransfusion after orthopaedic surgery; Healy W.; Pfeifer S.; Kurtz U.; Clin. Orthop., N. 299 (1994), 53-59.

29. Conservation of blood during cardiovascular surgery, Cooley D.A; American Journal of Surgery , 170 , N. 6A, Suppl. (1995), 53S-59S.

30. Reduction of blood loss and transfusion requirement by aprotinin in posterior lumbar spine fusion ; Lentschesner C.; Cottin H., Anesth. Analg. 89, N. 3, (1999), 590-597.

31. Central venous pressure and its effect on blood loss during liver resection ; Jones R.; Moulton M.; Br. J. Surg. 85 N. 8 (1998), 1058-1060.

32. Pharmacologic drugs for controlled hypotension; Testa L.D.; Tobias F.; j. Clin. Anesth., 7, N. 4 (1995),326-337.

33. Scott N. B. Regional anaesthesia and surgical morbidity. Br, J, Surg 1099; 75 (4): 44-p.

34. Grass J. Surgical outcome: regional anesthesia and analgesia versus general anaesthesia. Anesthesiol 1993; 20 (4): 117125.

35. Perioperative outcomes of major hepatic resections under low central venous pressure anesthesia: blood loss, blood transfusion, and the risk of postoperative renal dysfunction; J. Am. Coll. Surg 187, N. 6 (1998);620-625
36. Deliberate hypotensive anesthesia with labetalolin reconstructive surgery for scoliosis, Acta Anaesthesiol Sin, 34 N. 4; (1996); 203-207.

37. The role of desmopressin in reducing blood loss during lumbar fusions; Johnson R.; Murphy U.; Surg. Gynecol. Obstet.; 171, n. 3 (1990), 106-110.

38. Prevention of bleeding after cardiopulmonary bypass with high - dose tranexamic acid; Karski Y.; Teasdale R., J. Thorac. Cardiovasc. Surg. ,110, N.3, (1995), 835-42.

39. Tranexamic acid is effective in decreasing postoperative bleeding and transfusions in primary coronary artery bypass surgery : a double blind randomized palcebo controlled trial; Brown G.; Thwaites R.; Anesth. Analg. 85; n.5 (1997), 963-70.

40. Increased safety in the administration of aprotinin, need for a test dose; Llau V.; García- Pérez M., Nesth. Analg. 90 , N. 3 (2000), 770-71.

41. Kesten S.A. Hoyas de C. Chaparrto I. Aprtinin reduced blood loss in lung transplant recipients Ann Thorac, Surg 1995; 59 (4): 877-879.

42. Lentschesner C, Cottin H. Reduction of blood loss and transfusion requirement by aprotinin in posterior lumbar spine fusion Anesth Analg 1999; 89 (3): 590-97.

43. aprotinin and transfusion requirements in orthoptic liver transplantation : a multicentric randomised double blind trial ; Porte Y., Moleenar T., Begliomini U.; Lancet, 335, N. 9212; (2000), 1303-1309.

44. Jaramillo M. Medicina Transfusional, Principios y practica medico quirúrgica. Bogotá: Proyecto Aventis, 2003.

45. Szalay D, Wong T, Lindsay T. Impact of red cell salvage on transfusión requirements during elective abdominal aortic aneurysm repair Ann. Vasc Surg 1999; 13 (6): 576-81.

46. Goodnough L. Monk G. Sicard H, J. Intraoperative salvage in patients undergoing elective abdominal aortic aneurysm reapir: an análisis of costs and benefits . J. Vasc. Surg 1996; 24 (2): 213-18.

47. Assesment of the availability of intraoperative autotransfusion in urological operations. J Urol 1997; 157 (5): 1777-80.

48. Heiss M, Anwar T. Delanoff C, Algayer H. Perisurgical erythropoietin application in anemic patients with colorectal cancer : A double - blind randomized study. Surgery 1996; my.

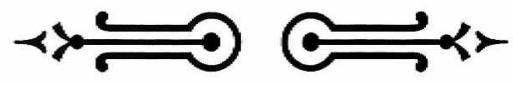

